\title{
Load Experiment of the vDACS Scheme in case of the 300 Clients
}

\author{
Kazuya Odagiri ${ }^{1}$, Shogo Shimizu ${ }^{2}$, Naohiro Is Iii $^{3}$ \\ ${ }^{1}$ Sugiyama Jogakuen University, \\ 17-3 Hosigaokamotomachi Chikusa-ku \\ Nagoya, Aichi 464-8662, Japan \\ E-mail:kodagiri@sugiyama-u.ac.jp,kazuodagiri@yahhoo.co.jp \\ ${ }^{2}$ Gakushuin Women's College, \\ 3-20-1 Toyama Shinzyuku-ku \\ Tokyo 162-8650, japan \\ E-mail: shogo.shimizu@gakushuin.ac.jp \\ ${ }^{2}$ Aichi Institute of Technology, \\ 1247 Yachigusa Yakusa-cho \\ Toyota Aichi 470-0392,japan \\ E-mail: ishii@aitech.ac.jp
}

\begin{abstract}
In the current Internet system, there are many problems using anonymity of the network communication such as personal information leaks and crimes using the Internet system. This is why TCP/IP protocol used in Internet system does not have the user identification information on the communication data, and it is difficult to supervise the user performing the above acts immediately. As a study for solving the above problem, there is the study of Policy Based Network Management (PBNM). This is the scheme for managing a whole Local Area Network (LAN) through communication control for every user. In this PBNM, two types of schemes exist. The first is the scheme for managing the whole LAN by locating the communication control mechanisms on the path between network servers and clients. The second is the scheme of managing the whole LAN by locating the communication control mechanisms on clients. As the second scheme, we have studied theoretically about the Destination Addressing Control System (DACS) Scheme. By applying this DACS Scheme to Internet system management, we will realize the policy-based Internet system management. In this paper, as the progression phase for the last goal, we perform the load experiment of the cloud type virtual PBNM named the vDACS Scheme, which can be used by plural organizations, for applications to the small and medium size scale organization. The number of clients used in an experiment is 300 .
\end{abstract}

\section{Introduction}

In the current Internet system, there are many problems using anonymity of the network communication such as personal information leaks and crimes using the Internet system. The news of the information leak in the big company is sometimes reported through the mass media. Because TCP/IP protocol used in Internet system does not have the user identification information on the communication data, it is difficult to supervise the user performing the above acts immediately. As studies and technologies for managing Internet system realized on TCP/IP protocol, those such as Domain Name System (DNS), Routing protocol, Fire Wall (F/W) and Network address port translation (NAPT)/network address translation (NAT) are listed. Except these studies, 
various studies are performed elsewhere. However, they are the studies for managing the specific part of the Internet system, and have no purpose of solving the above problems.

As a study for solving the problems, Policy Based Network Management (PBNM) [2] exists. The PBNM is a scheme for managing a whole Local Area Network (LAN) through communication control every user, and cannot be applied to the Internet system. This PBNM is often used in a scene of campus network management. In a campus network, network management is quite complicated. Because a computer management section manages only a small portion of the wide needs of the campus network, there are some user support problems. For example, when mail boxes on one server are divided and relocated to some different server machines, it is necessary for some users to update a client machine's setups. Most of computer network users in a campus are students. Because students do not check frequently their e-mail, it is hard work to make them aware of the settings update. This administrative operation is executed by means of web pages and/or posters. For the system administrator, individual technical support is a stiff part of the network management. Because the PBNM manages a whole LAN, it is easy to solve this kind of problem. In addition, for the problem such as personal information leak, the PBNM can manage a whole LAN by making anonymous communication nonanonymous. As the result, it becomes possible to identify the user who steals personal information and commits a crime swiftly and easily. Therefore, by applying the PBNM, we will study about the policybased Internet system management.

In the existing PBNM, there are two types of schemes. The first is the scheme of managing the whole LAN by locating the communication control mechanisms on the path between network servers and clients. The second is the scheme of managing the whole LAN by locating the communication control mechanisms on clients. It is difficult to apply the first scheme to Internet system management practically, because the communication control mechanism needs to be located on the path between network servers and clients without exception. Because the second scheme locates the communication control mechanisms as the software on each client, it becomes possible to apply the second scheme to Internet system management by devising the installing mechanism so that users can install the software to the client easily.

As the second scheme, we have studied theoretically about the Destination Addressing Control System (DACS) Scheme. As the works on the DACS Scheme, we showed the basic principle of the DACS Scheme, and security function [14]. After that, we implemented a
DACS System to realize a concept of the DACS Scheme. By applying this DACS Scheme to Internet system, we will realize the policy-based Internet system management. Then, the Wide Area DACS system (wDACS system) [15] to use it in one organization was showed as the second phase for the last goal. As the first step of the second phase, we showed the concept of the cloud type virtual PBNM, which could be used by plural organizations [16]. In this paper, as the progression phase of the third phase for the last goal, we perform the load experiment to confirm the possibility of the cloud type virtual PBNM for the use in plural organizations. In Section 2, motivation and related research for this study are described. In Section 3, the existing DACS Scheme and wDACS Scheme is described. In section4 the proposed scheme and load experiment results are described.

\section{Motivation and Related Reserach}

In the current Internet system, problems using anonymity of the network communication such as personal information leak and crimes using the Internet system occur. Because TCP/IP protocol used in Internet system does not have the user identification information on the communication data, it is difficult to supervise the user performing the above acts immediately.

As studies and technologies for Internet system management to be comprises of TCP/IP [1], many technologies are studied. For examples, Domain name system (DNS), Routing protocol such as Interior gateway protocol (IGP) such as Routing information protocol (RIP) and Open shortest path first (OSPF), Fire Wall (F/W), Network address translation (NAT) / Network address port translation (NAPT) , Load balancing, Virtual private network (VPN), Public key infrastructure (PKI), Server virtualization. Except these studies, various studies are performed elsewhere. However, they are for managing the specific part of the Internet system, and have no purpose of solving the above problems.

As a study for solving the above problem, the study area about PBNM exists. This is a scheme of managing a whole LAN through communication control every user. Because this PBNM manages a whole LAN by making anonymous communication non-anonymous, it becomes possible to identify the user who steals personal information and commits a crime swiftly and easily. Therefore, by applying this policy- based thinking, we study about the policy-based Internet system management. 


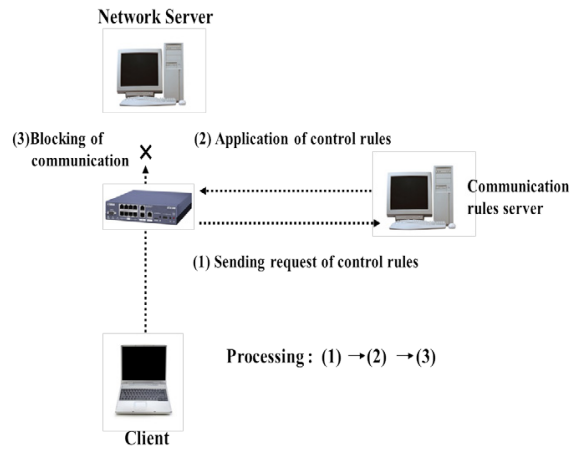

Figure 1. Principle in First Scheme

In policy-based network management, there are two types of schemes. The first scheme is the scheme described in Figure 1. The standardization of this scheme is performed in various organizations. In IETF, a framework of PBNM [2] was established. Standards about each element constituting this framework are as follows. As a model of control information stored in the server called Policy Repository, Policy Core Information model (PCIM) [3] was established. After it, PCMIe [4] was established by extending the PCIM. To describe them in the form of Lightweight Directory Access Protocol (LDAP), Policy Core LDAP Schema (PCLS) [5] was established. As a protocol to distribute the control information stored in Policy Repository or decision result from the PDP to the PEP, Common Open Policy Service (COPS) [6] was established. Based on the difference in distribution method, COPS usage for RSVP (COPS-RSVP) [7] and COPS usage for Provisioning (COPS-PR) [8] were established. RSVP is an abbreviation for Resource Reservation Protocol. The COPS-RSVP is the method as follows. After the PEP having detected the communication from a user or a client application, the PDP makes a judgmental decision for it. The decision is sent and applied to the PEP, and the PEP adds the control to it. The COPS-PR is the method of distributing the control information or decision result to the PEP before accepting the communication.

Next, in DMTF, a framework of PBNM called Directory-enabled Network (DEN) was established. Like the IETF framework, control information is stored in the server storing control information called Policy Server, which is built by using the directory service such as LDAP [9], and is distributed to network servers and networking equipment such as switch and router. As the result, the whole LAN is managed. The model of control information used in DEN is called Common Information Model (CIM), the schema of the CIM (CIM Schema Version 2.30.0) [11] was opened. The CIM was extended to support the DEN [10], and was incorporated in the framework of DEN.
In addition, Resource and Admission Control Subsystem (RACS) [12] was established in Telecoms and Internet converged Services and protocols for Advanced Network (TISPAN) of European Telecommunications Standards Institute (ETSI), and Resource and Admission Control Functions (RACF) was established in International Telecommunication Union Telecommunication Standardization Sector (ITUT) [13].

However, all the frameworks explained above are based on the principle shown in Figure 1. As problems of these frameworks, two points are presented as follows. Essential principle is described in Figure 2. To be concrete, in the point called PDP (Policy Decision Point), judgment such as permission and nonpermission for communication pass is performed based on policy information. The judgment is notified and transmitted to the point called the PEP, which is the mechanism such as VPN mechanism, router and Fire Wall located on the network path among hosts such as servers and clients. Based on that judgment, the control is added for the communication that is going to pass by.

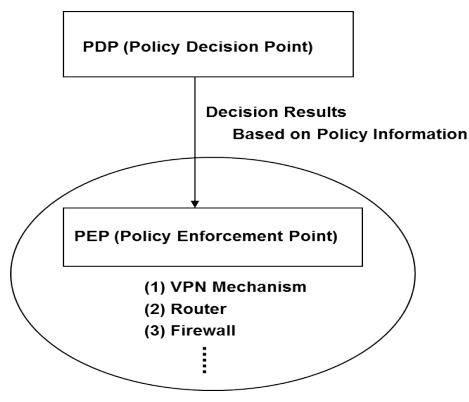

Figure 2. Essential Principle

The principle of the second scheme is described in Figure 3.By locating the communication control mechanisms on the clients, the whole LAN is managed. Because this scheme controls the network communications on each client, the processing load is low. However, because the communication control mechanisms need to be located on each client, the work load becomes heavy.

When it is thought that Internet system is managed by using these two schemes, it is difficult to apply the first scheme to Internet system management practically. This is why the communication control mechanism needs to be located on the path between network servers and clients without exception. On the other hand, the second scheme locates the communication controls mechanisms on each client. That is, the software for communication control is installed on each client. So, by devising the installing mechanism letting users install software to the client easily, it becomes possible to apply the second scheme to Internet system management. As a first step 
for the last goal, we showed the Wide Area DACS system (wDACS) system [15]. This system manages a wide area network, which one organization manages. Therefore, it is impossible for plural organizations to use this system.Then, as the first step of the second phase, we showed the concept of the cloud type virtual PBNM, which could be used by plural organizations in this paper.

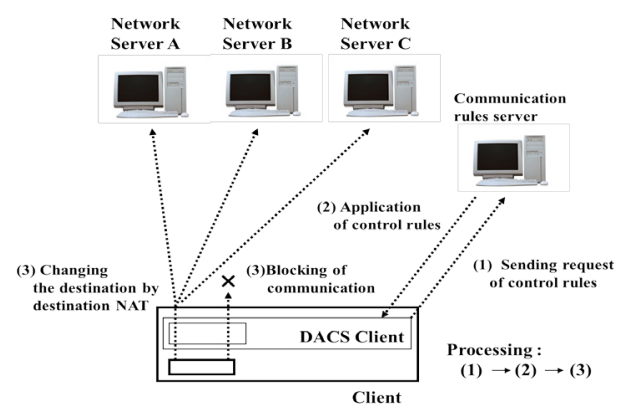

Figure 3. Principle in Second Scheme

\section{Existing DACS SCHEME and wDACS System}

In this section, the content of the DACS Scheme which is the study of the phase 1 is described.

\subsection{Basic Principle of the DACS Scheme}

Figure 4 shows the basic principle of the network services by the DACS Scheme. At the timing of the (a) or (b) as shown in the following, the DACS rules (rules defined by the user unit) are distributed from the DACS Server to the DACS Client.

(a) At the time of a user logging in the client.

(b) At the time of a delivery indication from the system administrator.

According to the distributed DACS rules, the DACS Client performs (1) or (2) operation as shown in the following. Then, communication control of the client is performed for every login user.

(1) Destination information on IP Packet, which is sent from application program, is changed.

(2) IP Packet from the client, which is sent from the application program to the outside of the client, is blocked.

An example of the case (1) is shown in Figure 4. In Figure 4, the system administrator can distribute a communication of the login user to the specified server among servers A, B or C. Moreover, the case (2) is described. For example, when the system administrator wants to forbid an user to use MUA (Mail User Agent), it will be performed by blocking IP Packet with the specific destination information.

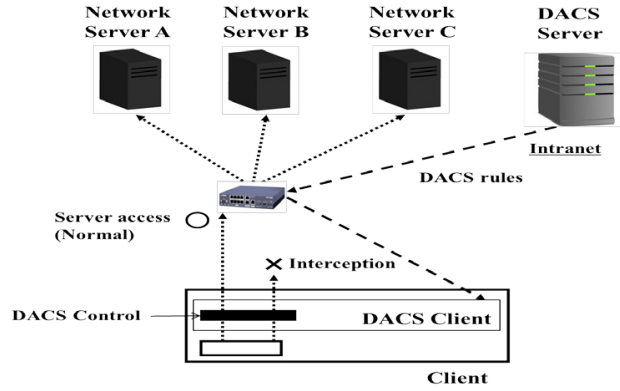

Figure 4. Basic Principle of the DACS Scheme

In order to realize the DACS Scheme, the operation is done by a DACS Protocol as shown in Figure 5. As shown by (1) in Figure 5, the distribution of the DACS rules is performed on communication between the DACS Server and the DACS Client, which is arranged at the application layer. The application of the DACS rules to the DACS Control is shown by (2) in Figure 5 .

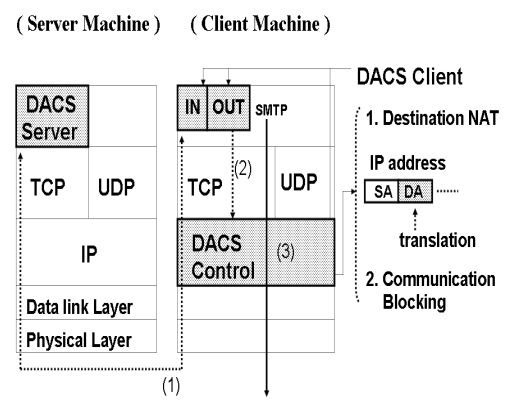

Figure 5. Layer Setting of the DACS Scheme

The steady communication control, such as a modification of the destination information or the communication blocking is performed at the network layer as shown by (3) in Figure 5.

\subsection{Application to cloud environment}

In this section, the contents of wDACS system are explained in Figure 6. First, as preconditions, because private IP addresses are assigned to all servers and clients existing in from LAN1 to LAN n, mechanisms of NAT/NAPT are necessary for the communication from each LAN to the outside. In this case, NAT/NAPT is located on the entrance of the LAN such as (1), and the private IP address is converted to the global IP address towards the direction of the arrow. Next, because the private IP addresses are set on the servers and clients in the LAN, other communications except those converted by Destination NAT cannot enter into the LAN. But, responses for the communications sent form the inside of the LAN can enter into the inside of the LAN 
because of the reverse conversion process by the NAT/NAPT. In addition, communications from the outside of the LAN1 to the inside are performed through the conversion of the destination IP address by Destination NAT. To be concrete, the global IP address at the same of the outside interface of the router is changed to the private IP address of each server. From here, system configuration of each LAN is described. First, the DACS Server and the authentication server are located on the DMZ on the LAN1 such as (4). On the entrance of the LAN1, NAT/NAPT and destination NAT exists such as (1) and (2). Because only the DACS Server and network servers are set as the target destination, the authentication server cannot be accessed from the outside of the LAN1. In the LANs form LAN 2 to LAN n, clients managed by the wDACS system exist, and NAT/NAPT is located on the entrance of each LAN such as (1). Then, F/W such as (3) or (5) exists behind or with NAT/NAPT in all LANs.

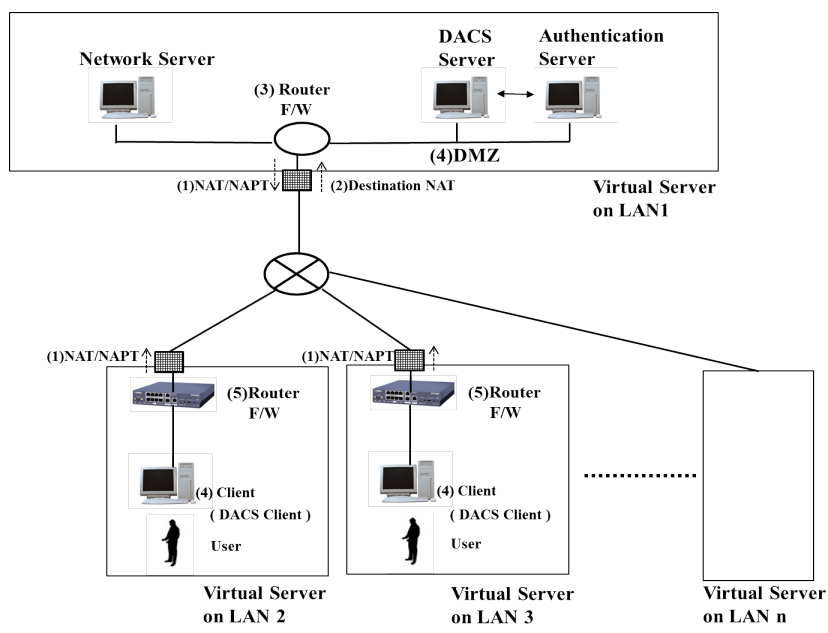

Figure 6. Basic System Configuration of wDACS system

\section{The Cloud Type Virtual PBNM for the common use between Plural organizations}

In this section, after the concept and implementation of the proposed scheme were described, functional evaluation results are described.

\subsection{Concept of the Cloud Type Virtual PBNM for the Common Use between Plural Organizations}

In Figure 7 which is described in [16], the proposed concept is shown. Because the existing wDACS Scheme realized the PBNM control with the software called the DACS Server and the DACS client, other mechanism was not needed. By this point, application to the cloud environment was easy.

The proposed scheme in this paper realizes the common usage by plural organizations by adding the following elements to realize the common usage by plural organizations: user identification of the plural organizations, management of the policy information of the plural organizations, application of the PKI for code communication in the Internet, Redundant configuration of the DACS Server (policy information server), load balancing configuration of the DACS Server, installation function of DACS Client by way of the Internet .

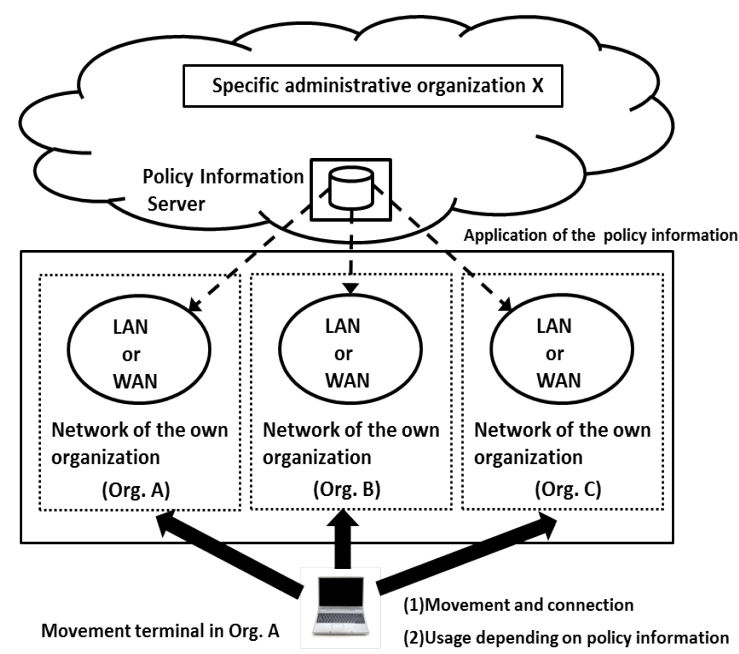

Figure 7. Concept of the proposed scheme

\subsection{Implementation of the basic function in the Cloud Type Virtual PBNM for the Common Usage between Plural Organizations}

In the past study [14], the DACS Client was operated on the windows operation system (Windows OS). It was because there were many cases that the Windows OS was used for as the OS of the client. However, the Linux operating system (Linux OS) had enough functions to be used as the client recently, too. In addition, it was thought that the case used in the clients in the future came out recently. Therefore, to prove the possibility of the DACS Scheme on the Linux OS, the basic function of the DACS Client was implemented in this study. The basic functions of the DACS Server and DACS Client were implemented by JAVA language. From here, it is described about the order of the process in the DACS Client and DACS Server as follows.

(Processes in the DACS Client)

(p1) The information acquisition from Cent OS

(p2) Transmission from the DACS Client to the DACS 
(p3) The information transmission from the DACS Client to

(p4) The reception of the DACS rules from the DACS Server

(p5) Application of the DACS rules of the DACS Control

(Processes in the DACS Server)

(p1) The information reception from the DACS Client

(p2) Connection to the database

(p3) Inquiry of the Database

(p4) Transmission of the DACS rules to the DACS Client

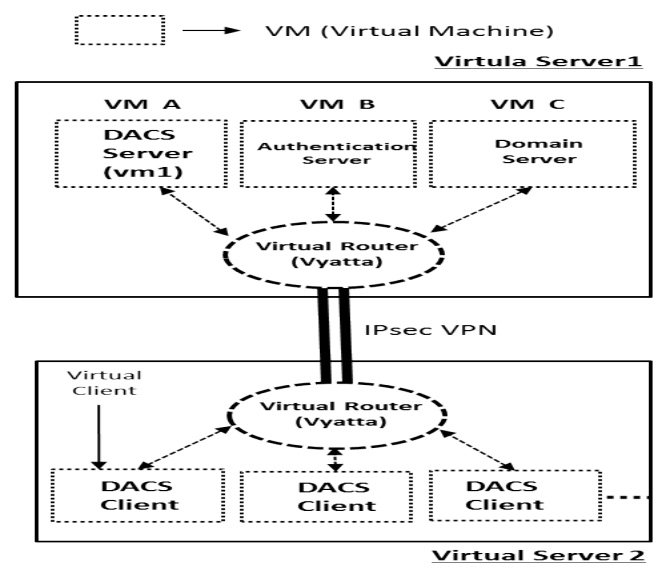

Figure.8 Prototype system

\subsection{Results of the functional evaluation}

In this section, the results of the functional evaluation for the implementation system are described in Figure 8.

In Figure 9, the setting situation of the DACS rules is described in figure 9. This DACS rules is the rule to change a Web server for the access. The delivery of the DACS rules is between the DACS SV and the DACS CL encrypted by using SSL.

By this DACS rules, the next operation was realized. When the user accessed the Web Server with the IP address of 192.168.1.10, the Web Server with the IP address of 192.168.1.12 was accessed actually. As for this communication result, the communication $\log$ on each Web server was confirmed by viewing.

〈?ML version="1.0" encoding="uft8"?

$\langle$ direct〉

〈rule priority="0" table=" nat" ipv="ipv4" chain="PREROUTING_di rect"〉 -d 192.168.1.10:80 - j DNGT --to 192.168.1.12:80</rule〉

$\langle/$ direct

Figure.9 Setting situation of the DACS rules on the DACS CL

\subsection{Load experiment results to confirm the function of the software for realization of the Cloud Type Virtual PBNM for the Common Use Between Plural Organizations}

In this section, the load experiment results are described. In the Figure 10, the experimental environment is described. This environment consists of four virtual servers. In the virtual server 1 , servers group such as the DACS Server and user authentication server is stored. In other virtual severs such as the virtual server 2 , virtual server 3 and virtual server 4 , the virtual client which is installed the DACS Client is stored. The number of the virtual clients is 100. By using this experimental environment, the load experiment was executed.

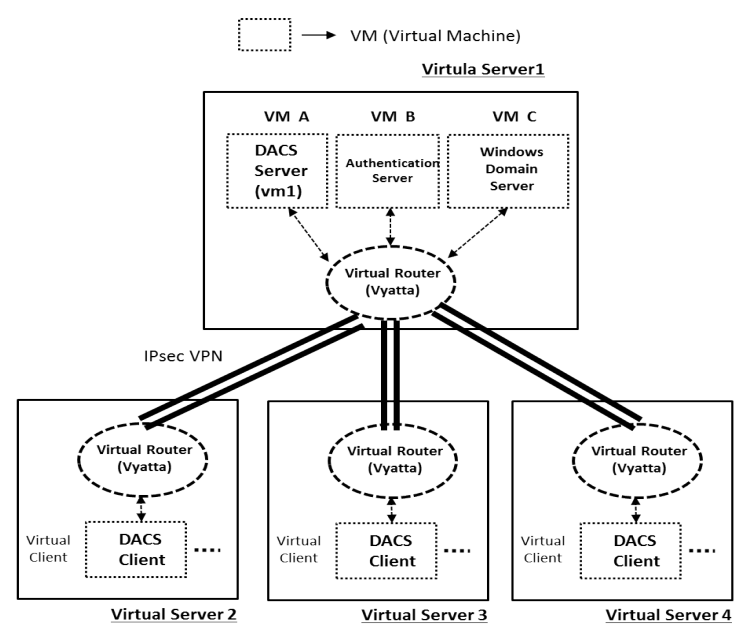

Figure.10 Experimental environment

(Experimental results by 100 clients)

The average of the results of the measurement for ten times was $263.2 \mathrm{MHz}$.

(Experimental results by 200 clients)

The average of the CPU consumption was $540.1 \mathrm{MHz}$. The value of around $540 \mathrm{MHz}$ is the CPU load when the 200 clients are connected simultaneously.

After these experiments, the load experiments by 300 clients were performed. The experimental environment is described. The experimental environment is as previous experiment environment. The results are described as follows.

(Case of 10 simultaneous connections at the DACS SV) In the first experiment, the number of the simultaneous connection for the DACS SV was set to 10 on this occasion. The experimental results are described in Figure 11. 


\begin{tabular}{|c|c|r|}
\hline & Practice Time & CPU Consumption \\
\hline $\mathbf{1}$ & $17: 00$ & 724 \\
\hline $\mathbf{2}$ & $17: 15$ & 724 \\
\hline $\mathbf{3}$ & $17: 30$ & 745 \\
\hline $\mathbf{4}$ & $17: 45$ & 730 \\
\hline $\mathbf{5}$ & $18: 00$ & 749 \\
\hline $\mathbf{6}$ & $18: 15$ & 737 \\
\hline $\mathbf{7}$ & $18: 30$ & 725 \\
\hline $\mathbf{8}$ & $18: 45$ & 754 \\
\hline $\mathbf{9}$ & $19: 00$ & 717 \\
\hline $\mathbf{1 0}$ & $19: 15$ & 723 \\
\hline
\end{tabular}

Figure.11 Experimental results (1)

In this Figure, the average of the results of the measurement for ten times was $732.8 \mathrm{MHz}$.

(Case of 20 simultaneous connections at the DACS SV)

In the second experiment, the number of the simultaneous connection for the DACS SV was set to 20 on this occasion. The experimental results are described in Figure 12.

\begin{tabular}{|c|c|r|}
\hline & Practice Time & CPU Consumption \\
\hline $\mathbf{1}$ & $20: 45$ & 766 \\
\hline $\mathbf{2}$ & $21: 00$ & 748 \\
\hline $\mathbf{3}$ & $21: 15$ & 763 \\
\hline $\mathbf{4}$ & $21: 30$ & 777 \\
\hline $\mathbf{5}$ & $21: 45$ & 785 \\
\hline $\mathbf{6}$ & $22: 00$ & 716 \\
\hline $\mathbf{7}$ & $22: 15$ & 753 \\
\hline $\mathbf{8}$ & $22: 30$ & 721 \\
\hline $\mathbf{9}$ & $22: 45$ & 744 \\
\hline $\mathbf{1 0}$ & $23: 00$ & 728 \\
\hline
\end{tabular}

Figure.12 Experimental results (2)

In this Figure, the average of the results of the measurement for ten times was $750.1 \mathrm{MHz}$.

(Case of 30 simultaneous connections at the DACS SV)

In the third experiment, the number of the simultaneous connection for the DACS SV was set to 20 on this occasion. The experimental results are described in Figure 13.

\begin{tabular}{|c|c|r|}
\hline & Practice Time & CPU Consumption \\
\hline $\mathbf{1}$ & $0: 15$ & 758 \\
\hline $\mathbf{2}$ & $0: 30$ & 767 \\
\hline $\mathbf{3}$ & $0: 45$ & 754 \\
\hline $\mathbf{4}$ & $1: 00$ & 743 \\
\hline $\mathbf{5}$ & $1: 15$ & 742 \\
\hline $\mathbf{6}$ & $1: 30$ & 756 \\
\hline $\mathbf{7}$ & $1: 45$ & 730 \\
\hline $\mathbf{8}$ & $2: 00$ & 752 \\
\hline $\mathbf{9}$ & $2: 15$ & 720 \\
\hline $\mathbf{1 0}$ & $2: 30$ & 766 \\
\hline
\end{tabular}

Figure.13 Experimental results (3)

In this Figure, the average of the results of the measurement for ten times was $748.8 \mathrm{MHz}$.

As the results of these experiments, the CPU consumption becomes approximately constant in the case of the number of the simultaneous connection from 20 to 30 . The value of around $750 \mathrm{MHz}$ is the CPU load when the 300 clients are connected simultaneously.

\subsection{Evaluation for these load experiment results}

In this section, the evaluation for these load experiments is executed. In Figure. 14, the transition of the processing load which was generated on the DACS SV side when the number of clients is increased on the premise that the number of simultaneous connections is 10 is shown.

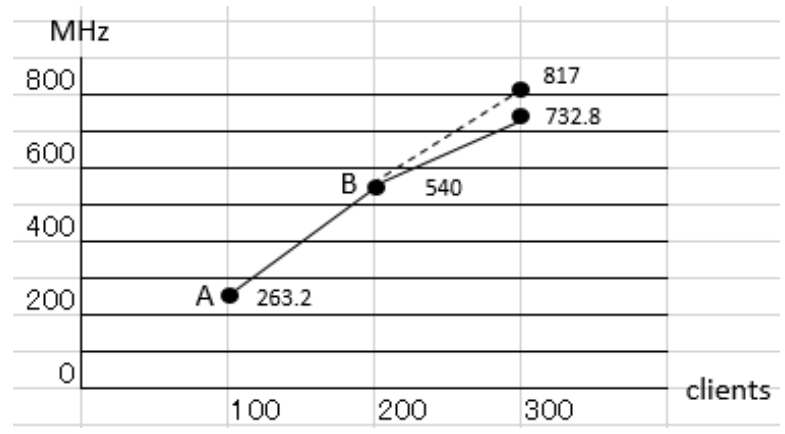

Figure.14 Transition in case of 10 simultaneous connections

In the graph this figure, the vertical axis shows the consumption of CPU, and the horizontal axis shows the number of clients. When the straight line connecting point $\mathrm{A}$ and point $\mathrm{B}$ is extended, it is a straight line like a dotted line. When the number of clients is 300 , the value of the $\mathrm{y}$ axis component of the point on the straight line of the dotted line is $817(\mathrm{MHz})$. However, the actual value is $732.8(\mathrm{MHz})$. Several causes are considered as causes of the difference. One possibility is that the CPU consumption of the virtual machine in which the DACS SV is arranged has reached $100 \%$. However, since it is confirmed that the CPU consumption is not $100 \%$ during the measurement experiment, this is not the cause.

What is considered as the next cause is the possibility that the processing load is adjusted on the virtual machine side. Regarding this point, even if the number of clients is 100 or 200 , the same possibility can be considered. However, even if some degree of automatic adjustment is performed, it can be evaluated that there are no problems with about 300 clients in case of .10 simultaneous connections.

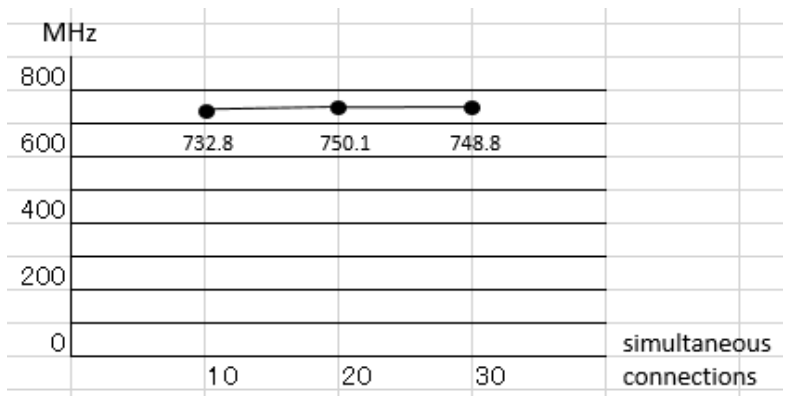

Figure.15 Transition by change of simultane ous connections in 300 clients 
In Figure.15, the transition of change in CPU consumption on the DACS SV side when the number of simultaneous connections was changed is shown. When the number of simultaneous connections is 10 , the CPU consumption on the DACS SV side is about $730 \mathrm{MHz}$. On the other hand, when the number of simultaneous connections is 20 or 30 , the CPU consumption on the DACS SV side is about $750 \mathrm{MHz}$. When compared with the value when the number of simultaneous connections is 10 , it is about 1.27 times. When the number of simultaneous connections was increased from 20 to 30 , no increase in value was observed. If the number of simultaneous connections is increased to 30 or more, it is expected that there will be no increase in value. Based on these experimental results, when 300 clients are managed by this proposed method, the CPU consumption on the DACS SV side is about $750 \mathrm{MHz}$. From the viewpoint of processing load, in a small organization, it is sufficient to configure the DACS SV with one virtual machine. However, in terms of fault tolerance in business continuity, redundancy of DACS $\mathrm{SV}$ is required.

\section{Conclusion}

In this paper, we performed the load experiment of the cloud type virtual PBNM, which can be used by plural organizations. In this experiment, the 300 virtual clients with Linux OS are used, and the communications between the DACS SV and the DACS CL are encrypted. As the result, the average of CPU consumption was $750.1 \mathrm{MHz}$. When the number of the simultaneous connection for the DACS SV was set to 30 on this occasion, the average of CPU consumption was 748.8 MHz. These two values are 2.8 times as large as the value in case of the 10 simultaneous connections.

As a future work, we are going to perform more load experiments in the form of increasing the number of the virtual client and the number of the simultaneous connection for the DACS SV.

\section{Acknowledgements}

This work was supported by the research grant of KDDI Foundation. We express our gratitude

\section{References}

1. V. CERF and E. KAHN, A Protocol for Packet Network Interconnection, IEEE Trans. on Commn 22(5) (1974)637-648.

2. R. Yavatkar, D. Pendarakis and R. Guerin, A Framework for Policy-based Admission Control, IETF RFC 2753 (2000).

3. B. Moore at el., Policy Core Information Model -Version 1 Specification, IETF RFC 3060 (2001).

4. B. Moore.,Policy Core Information Model (PCIM) Extensions, IETF 3460 (2003).

5. J. Strassner, B. Moore, R. Moats, E. Ellesson, Policy Core Lightweight Directory Access Protocol (LDAP) Schema, IETF RFC 3703 (2004).

6. D. Durham at el.,The COPS (Common Open Policy Service) Protocol, IETF RFC 2748 (2000).

7. S. Herzog at el.,COPS usage for RSVP, IETF RFC 2749 (2000).

8. K. Chan et al.,COPS Usage for Policy Provisioning (COPS-PR), IETF RFC 3084 (2001).

9. CIM Core Model V2.5 LDAP Mapping Specification (2002).

10. M. Wahl, T. Howes, S.Kille, Lightweight Directory Access Protocol (v3), IETF RFC 2251 (1997).

11. CIM Schema: Version 2.30 .0 (2011).

12. ETSI ES 282 003: Telecoms and Internet converged Services and protocols for Advanced Network (TISPAN); Resource and Admission Control Subsystem (RACS); Functional Architecture, (2006).

13. ETSI ETSI ES 283 026: Telecommunications and Internet Converged Services and Protocols for Advanced Networking (TISPAN); Resource and Admission Control; Protocol for QoS reservation information exchange between the Service Policy Decision Function (SPDF) and the Access-Resource and Admission Control Function (A-RACF) in the Resource and Protocol specifica-tion", (2006).

14. K. Odagiri, R. Yaegashi, M. Tadauchi, and N. Ishii, Secure DACS Scheme, Journal of Network and Computer Applications, Elsevier 31(4) (2008) 851-861.

15. K. Odagiri, S. Shimizu,M. Takizawa and N. Ishii, Theoretical Suggestion of Policy-Based Wide Area Network Management System (wDACS system part-I), International Journal of Networked and Distributed Computing (IJNDC) 1(4) (2013) 260-269.

16. K. Odagiri,S. Shimizu, N. Ishii, M. Takizawa, Suggestion of the Cloud Type Virtual Policy Based Network Management Scheme for the Common Use between Plural Organizations, in Proc of Int. Conf. on International Conference on Network-Based Information Systems (NBiS-2015)(Taipei, Taiwan, 2015),pp.180-186. 